\title{
Phase-transition Phenomena in Colloidal Systems with Attractive and Repulsive Particle Interactions
}

\author{
Agienus Vrij, ${ }^{*}$ Marcel H. G. M. Penders, Piet W. Rouw, Cornelis G. de Kruif, \\ Jan K. G. Dhont, Carla Smits and Henk N. W. Lekkerkerker \\ Van't Hoff laboratory, University of Utrecht, Padualaan 8, $3584 \mathrm{CH}$ Utrecht, \\ The Netherlands
}

\begin{abstract}
We discuss certain aspects of phase transitions in colloidal systems with attractive or repulsive particle interactions. The colloidal systems studied are dispersions of spherical particles consisting of an amorphous silica core, coated with a variety of stabilizing layers, in organic solvents. The interaction may be varied from (steeply) repulsive to (deeply) attractive, by an appropriate choice of the stabilizing coating, the temperature and the solvent. In systems with an attractive interaction potential, a separation into two liquidlike phases which differ in concentration is observed. The location of the spinodal associated with this demixing process is measured with pulseinduced critical light scattering. If the interaction potential is repulsive, crystallization is observed. The rate of formation of crystallites as a function of the concentration of the colloidal particles is studied by means of timeresolved light scattering.
\end{abstract}

Colloidal systems exhibit phase transitions which are also known for molecular/atomic systems. In systems consisting of spherical Brownian particles, liquid-liquid phase separation and crystallization may occur. Also gel and glass transitions are found. Moreover, in systems containing rod-like Brownian particles, nematic, smectic and crystalline phases are observed. A major advantage for the experimental study of phase equilibria and phase-separation kinetics in colloidal systems over molecular systems is the length- and time-scales that are involved. In colloidal systems length-scales are such that light scattering is an appropriate technique to probe microstructures and timescales are so large that it is fairly easy to perform meaningful time-resolved experiments during a phase transition. It is thus feasible to gain an understanding on the microscopic level of phase equilibria and phase-separation kinetics using light scattering on colloidal systems.

For colloidal systems it is possible to vary the pair-interaction potential more or less systematically. This can be done in several ways. As is well known, in the case of charged colloidal particles in water, the interaction potential can be varied by the addition of salt. For colloidal particles in apolar organic solvents on the surface of which polymer chains are grafted, the pair-interaction potential can be varied by either choosing a different polymer chain, changing the solvent or varying the temperature. In mixtures of apolar and polar solvents particles may be slightly charged, giving rise to a screened Coulomb type of interaction potential.

There is one complication in the comparison of results for phase-transition kinetics in colloidal systems and in molecular systems. In colloidal systems hydrodynamic interactions will influence the phase-separation kinetics. Since these hydrodynamic interactions are not pair-wise additive, a theoretical treatment of phase-separation kinetics in colloidal systems at higher volume fractions will be extremely difficult. At larger volume fractions it is therefore probably more sensible to predict general trends rather than to try to calculate experimentally measurable kinetic parameters in detail. 
In this paper we describe some of our experimental observations concerning the location of the spinodal and crystallization of colloidal systems. The location of the spinodal is measured using pulse-induced critical scattering (PICS). The kinetics of crystallization are studied using a specially designed light-scattering apparatus which enables us to perform time-resolved experiments.

\section{Attractive and Repulsive Interactions in Colloidal Systems}

In colloidal systems consisting of particles stabilized by a protective layer of (long-) chain molecules and having a particle core composed of material with a refractive index comparable to that of the solvent, the van der Waals-London attraction forces between the cores are small. ${ }^{1}$ The interaction forces are therefore dominated by the chain-chain and chain-solvent interactions of the opposing protective layers of the touching particle surfaces, which may range from repulsive to attractive depending on the 'quality' of the solvent.

Interactions due to protective layers of long-chain molecules were already investigated a long time ago. ${ }^{2}$ Studies on layers of short linear $\mathrm{C}_{18}$-alkane chains were reported for repulsive ${ }^{3}$ and more recently also for attractive forces and the transition between them. ${ }^{3-5}$

In view of the close packing of the stabilizing layers grafted onto the surface, two particles can hardly interpenetrate without imposing appreciable steric hindrance on the terminal chain segments. As a consequence, the interaction potential must become repulsive even at small overlap. Also the solvent molecules play a role here through 'solvation' interactions. If there is a preferential solvation of chain (ends) by solvent molecules, repulsive forces will already be felt before the bare chain segments are actually in contact, because the removal of solvent molecules requires work. The interparticle distance where the repulsive force becomes large is much larger than the thickness of the overlap region. Therefore these forces can be described as a hard-sphere repulsion, characterized by a hard-sphere diameter, whenever the chain molecules are small in comparison with the particle core diameter. This has turned out to be a very adequate model potential for dispersions of our silica particles in cyclohexane. ${ }^{6,7}$ For longer polymer chains the repulsion is softer. This feature plays an important role in the kinetics of crystallization in these systems. ${ }^{8}$

If, however, there is a preferential solvation of chain segments by other chain segments instead of solvent molecules, effective attraction forces result when two opposing particle surface layers approach each other. The details of these contributions must come from proper averaging of solvation forces as calculated ${ }^{9}$ and measured ${ }^{10,11}$ with smooth macroscopic surfaces. These attractive interactions may give rise to spinodal decomposition.

\section{Attractive Interactions and Demixing}

To describe attractions due to a preferential solvation of chain segments by other chain segments, an entropic and an energetic component to the local mixing process of chain segments and solvent molecules, by analogy with the Flory and Krigbaum model ${ }^{12}$ for polymer segments, are taken into account. For the interaction potential $V(r)$ between two particles the following form is used,

$$
V(r) / k_{\mathrm{B}} T=-L(r)\left(\frac{\theta}{T}-1\right) ; \quad T<\theta .
$$

Here $k_{\mathrm{B}}$ is Boltzmann's constant, $T$ is the temperature, $L(r)$ is proportional to the overlap volume of the polymer-coating layer at interparticle separation $r$ and $\theta$ is the theta 


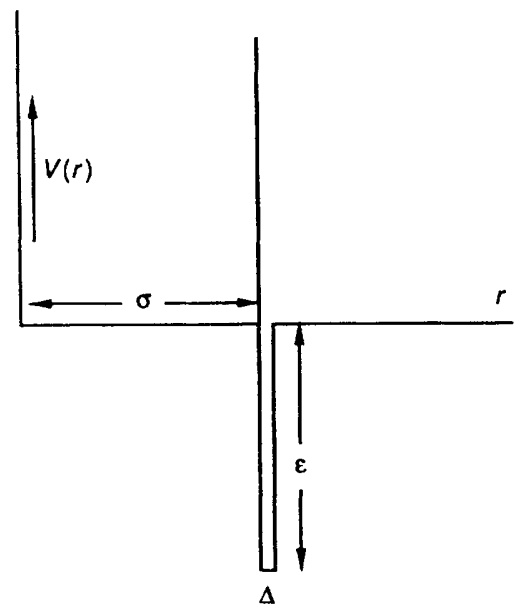

Fig. 1. The square-well interaction potential. The width of the well is $\Delta$, its depth is $\varepsilon$ and the hard-core diameter is $\sigma$.

temperature of the chain/solvent combination. The function $L(r)$ is non-zero in a short $r$ range only. For a good solvent $\theta \ll T$, and $V(r)$ is strongly positive. This implies that the bare hard-sphere diameter is just slightly increased, as mentioned before. In a poor solvent $\theta \gg T$. For $\theta>T, V(r)$ is an attractive well in a small range where $L(r)$ is non-zero. The attractive well disappears for $T \geqslant \theta$.

The question now arises as to which model potential may be used adequately to describe such a supramolecular fluid of adhesive hard spheres. Since it may be expected that, because of the narrowness of the attractive well, the actual shape of the well will not be very important, one may choose the potential that renders the simplest statistical thermodynamic connection between microscopic and macroscopic properties. Several model potentials consisting of a steep repulsion followed by a short-range attraction are possible. For a physically realistic potential, at least three model parameters are needed: the diameter of the hard core and the depth and range of the attractive well. A commonly used model fulfilling these requirements is the square-well potential, defined as,

$$
\begin{array}{rlrl}
V(r) & =\infty ; & r & <\sigma \\
& =-\varepsilon ; & & \sigma \leqslant r<\sigma+\Delta \\
& =0 ; & \sigma+\Delta \leqslant r
\end{array}
$$

This potential is sketched in fig. 1 . Here $\sigma$ is the hard-core diameter, $\varepsilon \geqslant 0$ is the depth of the well and $\Delta$ is the range or width of the well.

A relatively straightforward characterization of the interaction forces can be obtained from the osmotic pressure derivative $\partial \Pi / \partial \rho$,

$$
\frac{1}{k_{\mathrm{B}} T} \frac{\partial \Pi}{\partial \rho}=1+2 B_{2} \rho+\cdots
$$

where,

$$
B_{2}=2 \pi \int_{0}^{\infty} \mathrm{d} r r^{2}\left[1-\exp \left(-\frac{V(r)}{k_{\mathrm{B}} T}\right)\right] .
$$

Here $\Pi$ is the osmotic pressure, $\rho$ is the particle number density and $B_{2}$ is the second 
virial coefficient. In the case of the square-well model [eqn (2)] it is easily seen that,

$$
B_{2}=\frac{2}{3} \pi \sigma^{3}\left(1-\frac{\alpha}{8}\right)
$$

where

$$
\alpha=24 \frac{\Delta}{\sigma}\left[\exp \left(\frac{\varepsilon}{k_{\mathrm{B}} T}\right)-1\right]
$$

It follows that

$$
\frac{1}{k_{\mathrm{B}} T} \frac{\partial \Pi}{\partial \rho}=1+(8-\alpha) \phi+\cdots
$$

where $\phi=(\pi / 6) \rho \sigma^{3}$ is the volume fraction of hard-core material. The second virial coefficient is a difficult object to measure experimentally because one must perform experiments on dilute solutions with a volume fraction of say $<0.05$. Static lightscattering measurements, for example, are just not accurate enough for this goal. Fortunately, however, $\alpha$ can also be deduced from measurements of the particle diffusion coefficient. Diffusion coefficients from dynamic light-scattering measurements do not require inaccurate absolute intensity measurements. For the diffusion coefficient it is found that ${ }^{5,13,14}$

$$
\frac{D}{D_{0}}=1+k_{\mathrm{D}} \phi+\cdots
$$

with

$$
k_{D}=1.45-0.56 \alpha=1.45-13.44 \frac{\Delta}{\sigma}\left[\exp \left(\frac{L}{T}(\theta-T)\right)-1\right] .
$$

Here $D_{0}$ is the diffusion coefficient at infinite dilution. It should be mentioned that this simple relationship for $\alpha$ applies only for a 'sticky' hard sphere, i.e. a very short-range attractive well preceding the hard-core repulsion. As was mentioned before, this is the case in the systems used here. At each volume fraction the proportionality constant $k_{\mathrm{D}}$ in eqn (8) contains a constant term and an additive temperature-dependent term of the exponential type. A plot of the function $a(T)$, defined as

$$
a(T)=k_{\mathrm{B}} T / 6 \pi \eta(T) D(T)
$$

versus the temperature $T$ is plotted in fig. 2 for a stearyl silica coded SP23, in benzene. These particles have a hydrodynamic radius of $32 \mathrm{~nm}$. The three curves correspond to three volume fractions $0.0128,0.024$ and 0.0355 . In eqn $(10) \eta(T)$ is the temperaturedependent viscosity of the solvent. The displayed curves show a common point of intersection and a steep increase in a small temperature range of a few degrees around $T=310 \mathrm{~K}$. The point of intersection is located at a temperature where no net interactions are felt. According to eqn (9) this happens for $\alpha=1.465 / 0.56$. The increase of $a(T)$ is due to the rising attraction forces. The steepness of the curve is in fact consistent with the exponential dependence given in eqn (9). The drawn lines were calculated ${ }^{4}$ assuming $\Delta=0.3 \mathrm{~nm}$, and using $L$ and $\theta$ as fitting parameters; $L=94.7$ and $\theta=322.4 \mathrm{~K}$. Other somewhat arbitrary choices of $\Delta$ lead to similar values of $L$ and $\theta$. If $\Delta$ is changed from 0.1 to $0.9 \mathrm{~nm}, L$ changes from 96 to 90 and $\theta$ changes from 326 to $319 \mathrm{~K}$. Below a certain temperature threshold the value of $a(T)$ suddenly drops because of actual phase separation of the silica dispersion into a concentrated and a dilute phase.

Jansen ${ }^{15}$ found in his phase-separation studies that often the concentration of silica particles in the concentrated phase decreased with decreasing temperature in contradiction with theoretical predictions based on a simple gas-liquid type of phase transition. 


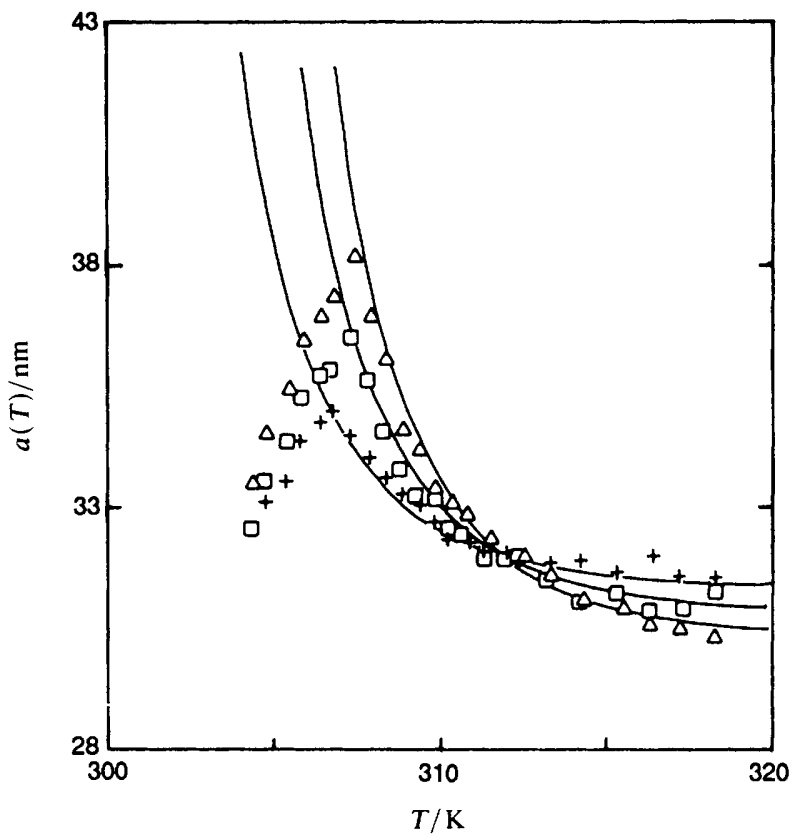

Fig. 2. The quantity $a(T)$, defined in eqn (10), as a function of the temperature for three volume fractions:,$+ 0.0128 ; \square, 0.0240 ; \triangle, 0.0355$.

He found the trend that the lower $T$ is, the lower the particle concentration becomes. The phases often had a gel-like appearance. It is for this reason that we started a new investigation of instability against phase separation by looking at states where the system is not yet phase-separated, but still homogeneous, i.e. by observing the spinodal curve with the technique of pulse-induced critical scattering. The technique is well known in the polymer field and is essentially a measurement of the critical light scattering upon fast cooling of the sample before any phase separation has occurred.

In fig. 3 the spinodal temperature $v s$. the volume fraction is plotted for the silica coded SJ4 in benzene (these particles have a hydrodynamic radius of $25 \mathrm{~nm}$ ). An important conclusion that can be drawn from this plot is that the measured spinodal curve has a 'parabolic' shape, in agreement with theoretical predictions of spinodal curves of the liquid-gas type. Thus no anomalous shape is found here. The drawn line is calculated using the so-called adhesive hard-sphere (AHS) or sticky hard-sphere model. This liquid-state model has been investigated with MC simulations ${ }^{16,17}$ and seems especially suited for particles with a very narrow attractive well as in our silica dispersions. The AHS model is a limiting case of a narrow attractive well at a hard-sphere surface, and was introduced by Baxter ${ }^{18}$ because it allows an exact solution of the approximate integral equation of Percus and Yevick for the liquid state. At the spinodal temperature the isothermal compressibility $\partial \rho / \partial \Pi$ goes to infinity. For the AHS model this means that,

$$
\left(\frac{\partial \Pi}{\partial \rho}\right)_{T}=\frac{(1-\phi)^{4}}{\left(1+2 \phi-\lambda_{B} \phi\right)^{2}}=0
$$

with

$$
\lambda_{\mathrm{B}}=6\left\{1+\frac{1-\phi}{\phi} \tau_{\mathrm{B}}-\sqrt{ }\left[\left(1+\frac{1-\phi}{\phi} \tau_{\mathrm{B}}\right)^{2}-\frac{1}{3}\left(\frac{1+\phi / 2}{\phi}\right)\right]\right\} .
$$




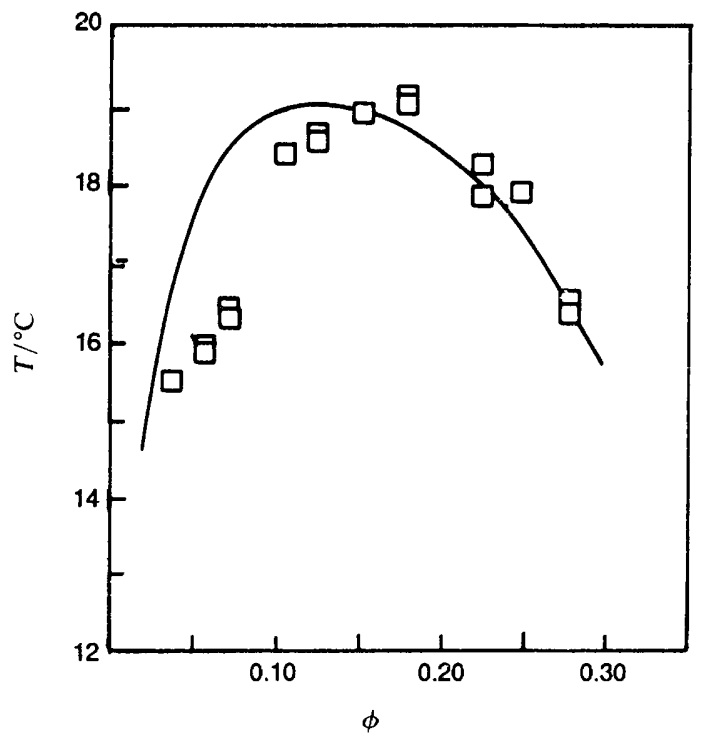

Fig. 3. The experimental and calculated AHS spinodal curve.

Here $\tau_{\mathrm{B}}$ is a stickiness parameter which is equal to $2 / \alpha$ and is related to the temperature according to eqn (6). The line drawn in fig. 3 is calculated using eqn (1), (2), (6), (11), (12), with $L=23.1$ and $\theta=355 \mathrm{~K}$, used as fitting parameters. The agreement between the experimental and theoretical spinodal in fig. 3 is quite reasonable.

\section{Repulsive Interactions and Crystallization}

In recent years crystallization has been observed in colloidal systems of sterically stabilized particles in organic solvents. It appears that the rate of crystallization is strongly dependent on the concentration ${ }^{19}$ and the rate of sedimentation. ${ }^{20-22}$ Also the nature of the stabilizing coating plays an important role. ${ }^{8}$ By coating the silica cores with different polymer chains a more or less soft repulsive potential can be realized in addition to a hard-core repulsion. We found that the rate of crystallization is extremely sensitive to the steepness of the repulsive potential. ${ }^{8}$ The observations described in ref. (8) are qualitative.

In this section we describe the first experiments in a quantitative light-scattering study of the kinetics of crystallization. The system on which these experiments are performed is a silica coated with a thin layer of $\gamma$-methacryloxypropyltrimethoxysilanesilica $^{23}$ (TPM-silica). The solvent is a mixture of ethanol and toluene with a composition such that its refractive index matches that of the particles. The particles are slightly charged, giving rise to a screened Coulomb type of interaction potential. The Debye length is estimated to be $c a .100 \mathrm{~nm} .^{24}$ The hard-core radius is $160 \mathrm{~nm}$.

After the chemical synthesis and purification of the particles, a dilute dispersion in ethanol-toluene is prepared, which is then centrifuged. The glassy sediment is redispersed, using a vortex mixer. Immediately after the redispersion, light-scattering curves are recorded. After completion of the crystallization process we found that shaking the sample with a vortex mixer, to within experimental error, the same nucleation/crystallization growth curve was reproduced as that recorded right after redispersion of the centrifuged sample. Contrary to vortex mixing, sonification of samples was found to lead to immediate crystallization. Apparently, sonification does not destroy crystallites, 


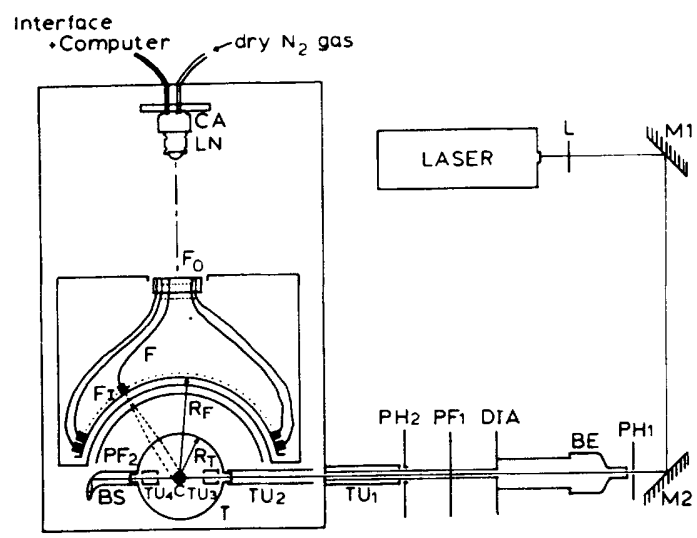

Fig. 4. A sketch of the static light-scattering apparatus. M1 and M2 are two mirrors used to align the laser beam. $L$ is a lens to make the beam parallel. PH1 and PH2 are two pinholes with a diameter of $3 \mathrm{~mm}$. BE is a beam expander and DIA a diaphragm which can be used to adjust the scattering volume. TU1-4 are beamguides to avoid any light other than the scattered light from the sample being detected. BS is a beamstop and PF1 is a polarization filter. PF2 is a circular sheet of polarization filter which is positioned in front of the optical-fibre channel inlets FI. $R_{\mathrm{T}}$ and $R_{\mathrm{F}}$ are the radii of the thermostatting vat and the circular array of the fibre channel inlets, respectively. The thermostatting vat serves also as a lens to focus the scattered radiation onto the fibre-channel inlets. The fibre-channel outlets $\mathrm{FO}$ are imaged onto a diode camera $\mathrm{CA}$ by means of a Nikon objective, LN. The camera operates at $5^{\circ} \mathrm{C}$ and must be flushed with dry nitrogen to prevent condensation on the diode array chip.

but even seems to enhance their rate of formation. Vortex mixing was found to destroy the nuclei.

Light-scattering curves were recorded with an apparatus which is specially designed for time-resolved measurements. For the experimental study of the kinetics of phase transitions on a timescale of say $10 \mathrm{~s}$ or less, scattered intensities at all angles simultaneously are needed. There is just no time to scan the relevant scattering angles with a single detector. The principle of the light-scattering apparatus is sketched in fig. $4 .{ }^{25,26}$ The thermostatted toluene bath acts also as a lens as to focus the scattered light onto the inlets of 140 optical fibre channels. The fibre channel outlets are arranged in a rectangular array, which is imaged onto an array of 512 diodes. The time resolution of the diode array camera (model M1452A from EGG) is $10 \mathrm{~ms}$ and its dynamic range is $2^{14}$. The intensity of each fibre channel outlet spreads over two diodes. What is observed then is a series of 140 peaks on the diode array, where the area of each peak corresponds to the intensity which is scattered at the corresponding scattering angle.

The time evolution of the scattering curves of TPM-silica dispersions was recorded in this way at several overall concentrations. Three such experiments are shown in fig. 5 , for overall concentrations of $0.280(a), 0.294(b)$ and $0.329 \mathrm{~g} \mathrm{~cm}^{-3}(c)$. In fig. $5(a)$ the experimentally measured points are plotted in the first and the last scattering curve only. For all other scattering curves the actually measured points are not indicated for clarity, but just connected by straight lines. As can be seen from this figure, the relative scatter in the Bragg-peak height and the growth rate of the Bragg-peak intensity are both functions of the overall concentration. Apart from experimental errors, the scatter in the Bragg-peak height is due to the finite number of crystallites in the scattering volume (which is $0.12 \mathrm{~cm}^{3}$ ). The intensity over the Debye-Scherrer ring is not a constant, but varies depending on the number of crystallites in the scattering volume and their size. The relative standard deviation of the Bragg-peak height varied enormously with the overall concentration of colloidal particles. The scattering curves shown here are 

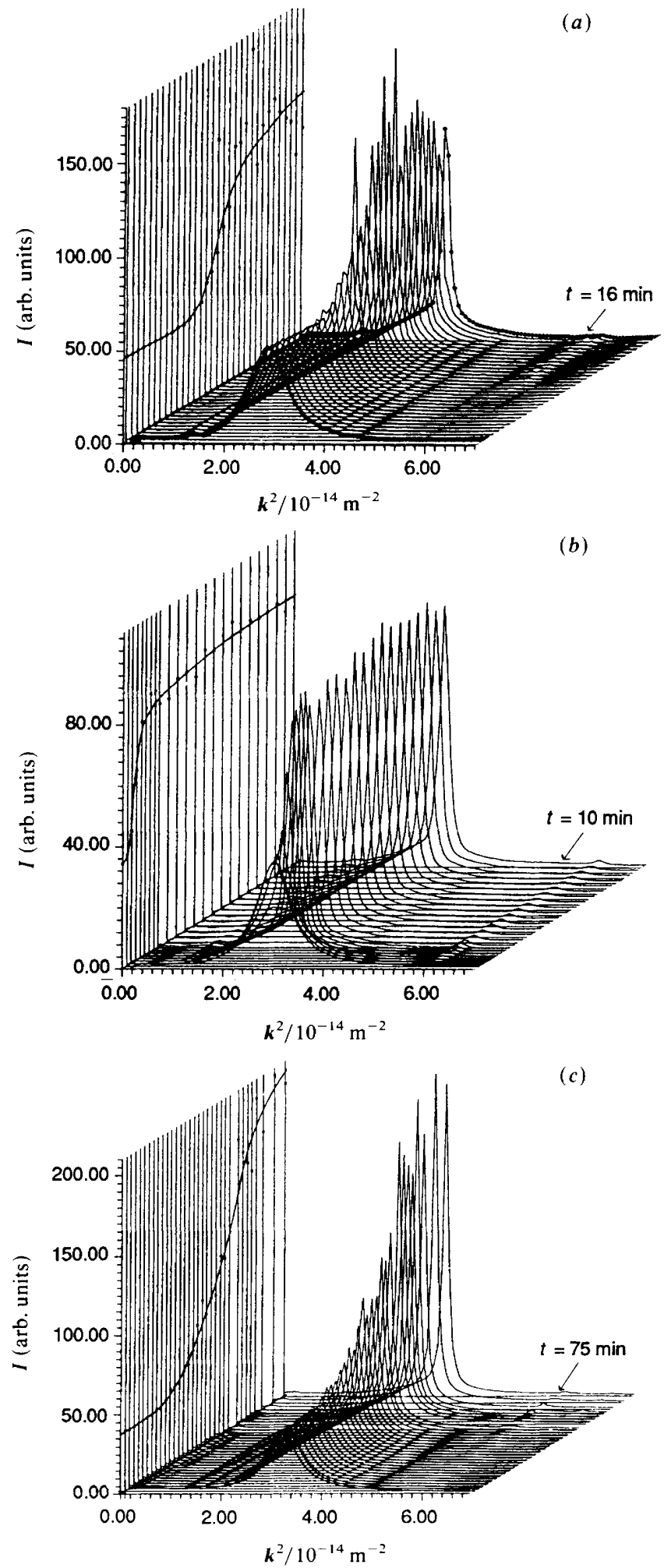

Fig. 5. Light-scattering curves for TPM silica at three overall concentrations: $(a) 0.280,(b) 0.294$ and $(c) 0.329 \mathrm{~g} \mathrm{~cm}^{-3} . I$ is the intensity in (for all figures the same) arbitrary units, $k$ is the scattering vector and $t$ is the time. The drawn line is the Bragg-peak height as a function of time. 


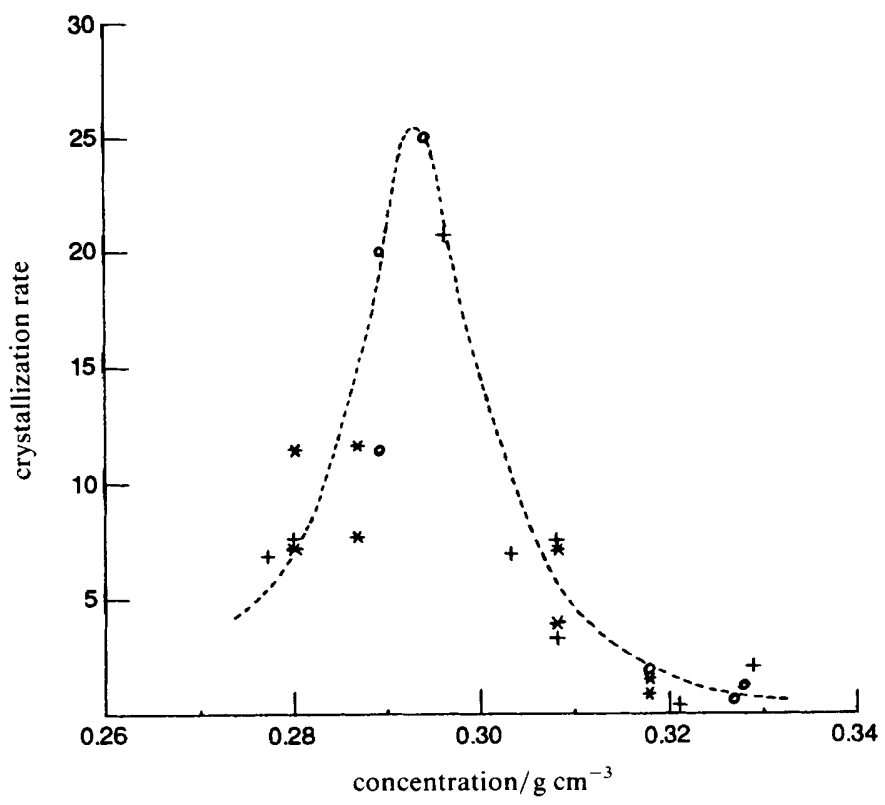

Fig. 6. The crystallization rate as a function of the overall concentration.

averages obtained by carefully rotating the cuvette during $12 \mathrm{~s}$, the time over which scattered intensities are collected. The actual variation of the intensity over the DebyeScherrer ring is therefore much larger than the scatter in the intensities in fig. 5 would suggest. The curves showing the Bragg-peak height vs. time are also given in fig. 5 as projections on the intensity $v s$. time plane. We have used the rate of increase of the Bragg-peak height as calculated from these curves as a measure for the crystallization rate. The crystallization rates thus determined, for several overall concentrations, are collected in fig. 6 . It is clear that the crystallization rate is a sensitive function of the overall concentration and displays a distinct maximum. Such a dependence was already observed qualitatively by Pusey and van Megen. ${ }^{19}$

The standard deviation in the Bragg-peak height for a given scattering volume contains both information on the size and the number concentration of crystallites in the sample. The width of the Bragg-peak is a measure for the size of the crystallites. We are currently measuring these quantities again as a function of the overall concentration. The scattering-angle resolution in these experiments is much better than in the experiments shown here (which was $1^{\circ}$ ).

\section{Summary}

In the colloidal systems consisting of spherical Brownian particles with an attractive pair-interaction potential, spinodal instabilities are observed. The location of the spinodal is measured with pulse-induced critical scattering. It is found that for the system of silica spheres with a short-range attractive potential that was used in our experiments, the location of the spinodal is well described by Baxter's adhesive hardsphere model.

Colloidal systems consisting of spherical particles with a soft repulsive pair-interaction potential crystallize at certain concentrations. The rate of crystallization as a function of the concentration shows, at least for the system under investigation in the 
present paper, one optimum within a narrow concentration range. We are currently investigating the number concentration and the size of the crystallites as a function of the concentration.

\section{References}

1 A. A. Caljé, W. G. M. Agterof and A. Vrii, in Micellization, Solubilization and Microemulsions, ed. K. L. Mittal (Plenum Press, New York, 1977), vol. 2.

2 D. H. Napper, in Polymer Stabilization of Colloidal Dispersions (Academic Press, London, 1983).

3 J. W. Jansen, C. G. de Kruif and A. Vrij, J. Colloid Interface Sci., 1986, 114, 471.

4 P. W. Rouw, C. G. de Kruif, J. Chem. Phys., 1988, 88, 7711.

5 R. Finsy, A. Devriese and H. N. W. Lekkerkerker, J. Chem. Soc., Faraday Trans. 2, 1980, 76, 767.

6 A. K. van Helden and A. Vrij, J. Colloid Interface Sci., 1980, 78, 312.

7 C. G. de Kruif, W. J. Briels, R. P. May and A. Vrij, Langmuir, 1988, 4, 668.

8 C. Smits, W. J. Briels, J. K. G. Dhont and H. N. W. Lekkerkerker, Prog. Colloid Polym. Sci., 1989, 79, 287.

9 D. Henderson, J. Colloid Interface Sci, 1988, 121, 486.

10 C. E. Herder, B. W. Ninham and H. K. Christenson, J. Chem. Phys., 1989, 90, 5801.

11 J. N. Israelachvili, S. J. Kott, M. K. Gee and T. A. Witten, Langmuir, 1989, 5, 1111.

12 P. J. Flory and W. R. Krigbaum, J. Chem. Phys., 1950, 18, 1086.

13 G. K. Batchelor, J. Fluid. Mech., 1972, 52, 245.

14 B. Cichocki and B. U. Felderhof, J. Chem. Phys., 1988, 89, 1049.

15 J. W. Jansen, C. G. de Kruif and A. Vrij, J. Colloid Interface Sci., 1986, 114, 481.

16 W. G. T. Kranendonk and D. Frenkel, Mol. Phys., 1988, 64, 403.

17 N. A. Seaton and E. D. Glandt, J. Chem. Phys., 1987, 87, 1785.

18 R. J. Baxter, J. Chem. Phys., 1968, 49, 2770.

19 P. N. Pusey and W. van Megen, Nature (London), 1986, 320, 340.

20 C. G. de Kruif, P. W. Rouw, J. W. Jansen and A. Vrij, J. Phys. C3., 1985, 46, 295.

21 S. Emmett, S. D. Lubetkin and B. Vincent, Colloid Surf., 1989, 42, 139.

22 K. E. Davis, W. B. Russel and W. J. Glantschnig, Science, 1989, 245, 507.

23 A. P. Philipse and A. Vrij, J. Colloid Interface Sci., 1989, 128, 121.

24 A. P. Philipse and A. Vrij, J. Chem. Phys., 1988, 88, 6459.

25 H. O. Moser, O. Fromhein, F. Herrmann and H. Versmold, J. Phys. Chem., 1988, 92, 6723.

26 J. K. G. Dhont, G. Harder, C. G. van der Werf, H. N. W. Lekkerkerker, H. Breiner and H. O. Moser, submitted. 